\title{
Exocomets in the circumstellar gas disk of HD 172555
}

\author{
F. Kiefer ${ }^{1,2}$, A. Lecavelier des Etangs ${ }^{1,2}$, J.-C Augereau $^{3}$, A. Vidal-Madjar ${ }^{1,2}$, A.-M. Lagrange ${ }^{3}$, and H. Beust ${ }^{3}$ \\ ${ }^{1}$ CNRS, UMR 7095, Institut d'Astrophysique de Paris, 98bis boulevard Arago, 75014 Paris, France \\ e-mail: kiefer@iap.fr \\ 2 UPMC Univ. Paris 6, UMR 7095, Institut d'Astrophysique de Paris, 98bis boulevard Arago, 75014 Paris, France \\ 3 UJF-Grenoble 1/CNRS-INSU, Institut de Planétologie et d'Astrophysique (IPAG) UMR 5274, 38041 Grenoble, France
}

Received 25 November 2013 / Accepted 30 December 2013

\section{ABSTRACT}

\begin{abstract}
The source HD 172555 is a young A7V star surrounded by a debris disk with a gaseous component. Here, we present the detection of variable absorption features detected simultaneously in the Ca II K and H doublet lines (at $\lambda 3933 \AA$ and $\lambda 3968 \AA$ ). We identified the presence of these absorption signatures at four different epochs in the 129 HARPS high-resolution spectra gathered between 2004 and 2011. These transient absorption features are most likely due to falling evaporating bodies (FEBs, or exocomets) that produce absorbing gas observed transiting in front of the central star. We also detect a stable Ca II absorption component at the star's radial velocity. With no corresponding detection in the $\mathrm{NaI}$ line, the resulting very low upper limit for the Na I/Ca II ratio suggests that this absorption is due to circumstellar gas.
\end{abstract}

Key words. planetary systems - stars: individual: HD 172555 - comets: general - circumstellar matter - ISM: clouds

\section{Introduction}

The source HD 172555 is an A7V-type star in the $\beta$ Pictoris moving group and harbors a dusty and gaseous circumstellar (CS) disk (Cote 1987; Lisse et al. 2009; Riviere-Marichalar et al. 2012). Lisse et al. (2009) and Johnson et al. (2012) have proposed that the dust was recently produced by a catastrophic collision between planetary mass bodies, which would have happened at around 5 au from the star. This scenario is consistent with the mid-infrared interferometric observations of the spatial distribution of dust detected at distances larger than 1 au from the central star (Smith et al. 2012). Up to now, the search for massive planets in the system have yielded only negative results (Quanz et al. 2011). The most striking recent discovery in this young circumstellar disk is the detection of [OI] emission at $63.2 \mu \mathrm{m}$ (Riviere-Marichalar et al. 2012), which shows that most of the mass of the disk must be in the gaseous phase. These works suggest that the combination of a dust debris disk with a gaseous circumstellar disk surrounding an A-type star a dozen million years old makes the HD 172555 system much resembling $\beta$ Pictoris.

The gaseous component of the $\beta$ Pic disk was detected in Ca II (Hobbs et al. 1985); it presents an anomalously low $\mathrm{NaI} / \mathrm{Ca}$ II ratio with a column density ratio $N(\mathrm{NaI}) /$ $N(\mathrm{Ca}$ II $) \sim 0.03$ and an equivalent width ratio $E q W\left(\mathrm{NaI} D_{2}\right) /$ $E q W(\mathrm{Ca}$ II $K) \sim 0.1$ (Vidal-Madjar et al. 1986). Furthermore, surveys of the $\beta$ Pic spectrum revealed the presence of variable and random additional absorptions, most often redshifted in the Ca II lines (Ferlet et al. 1987). These features are well interpreted in terms of falling evaporating bodies (FEBs, see review in Vidal-Madjar et al. 1998) or, in other words, exocomets.

With the $\beta$ Pic analogy in mind, we searched for CS gas and for any possible variable spectral signatures in the 129 spectra of HD 172555 collected from 2004 to 2011 using the HARPS spectrograph. We present the analysis of this data set in Sect. 2. A stable absorption component is detected in the Ca II doublet at the radial velocity of the star and is most likely of circumstellar origin (Sect. 3.1). We also present the detection of sporadic absorptions with typical characteristics of FEBs (Sect. 3.2). The results are discussed in Sect. 4.

\section{Data analysis}

The spectra were obtained from 2004 to 2011 with the HARPS spectrograph $(R \sim 115000)$ installed at the La Silla $3.6 \mathrm{~m}$ telescope (Table 2). We focused our attention on the Ca II stellar lines which are the most sensitive to the transit of gaseous clouds like the ones produced by exocomets in $\beta$ Pic. To ensure that any detected variation is of astrophysical origin and not due to variations in the wavelength or in the flux calibrations over the years, we compared the spectra in the region of the very steep $\mathrm{NaI}$ interstellar lines; this check confirms the tremendous stability of the instrument during the observation campaign (as expected for this spectrograph aimed at detecting minute variations in the star's radial velocities), and thus provides very high confidence to the reality of the detected spectral evolutions (see below).

From the 129 HARPS spectra, we calculated the reference spectrum $\left(F_{\text {ref }}\right)$ of HD 172555. It is used to search for variable absorption features and is obtained as follows. First, we assume that in the absence of variable absorption features the noise $\Delta F$ in the flux measurement is Gaussian, fluctuating around the real reference spectrum. We checked that its rms is proportional to $\sqrt{F}$ with a wavelength independent factor, for which we obtained a reliable estimation in FEB-free regions. Second, at each wavelength bin, the number $N$ of flux measurements is equal to the total number of spectra. Since we assumed that $\Delta F$ follows a Gaussian distribution, the $k$ th highest flux value $f_{k}$ is an estimator of the $\alpha_{k}$ rms level at which probability $P(\Delta F<$ $\alpha_{k}$ rms) is equal to $1-k / N$. By simple subtraction of $f_{k}$ from the $\alpha_{k}$ rms value we thus obtain an estimation $F_{\text {ref }, k}$ of the reference spectrum. The final reference spectrum is the mean of all these estimations obtained by varying $k$ from 3 to 10 .

In the case of $\beta$ Pic, this method has proved to be extremely robust for calculating the reference spectrum despite the 
A\&A 561, L10 (2014)

Table 1. Properties of the absorption features detected in the Ca II and Na I doublets. Radial velocities are given in heliocentric rest frame.

\begin{tabular}{|c|c|c|c|c|c|c|}
\hline \multirow[t]{2}{*}{$\begin{array}{l}\text { Line } \\
\text { origin }\end{array}$} & \multirow[t]{2}{*}{$\begin{array}{l}\text { Rad. vel. } \\
\left(\mathrm{km} \mathrm{s}^{-1}\right)\end{array}$} & \multicolumn{4}{|c|}{$\begin{array}{l}\text { Equivalent width } \\
(\mathrm{m} \AA)\end{array}$} & \multirow[t]{2}{*}{$\begin{array}{c}\mathrm{Na} / / \mathrm{Ca} \text { II ratio } \\
\left(\log _{10}(\mathrm{D} 2 / \mathrm{K})\right)\end{array}$} \\
\hline & & Ca II K & $\mathrm{Ca} I I \mathrm{H}$ & $\mathrm{Na} I \mathrm{D} 2$ & Na I D1 & \\
\hline IS & -19 & $0.87 \pm 0.08$ & $0.33 \pm 0.05$ & $1.02 \pm 0.05$ & $0.58 \pm 0.04$ & $0.07 \pm 0.10$ \\
\hline $\mathrm{CS}$ & 2 & $8.33 \pm 0.17$ & $4.66 \pm 0.12$ & $<0.31(5 \sigma)$ & $<0.31(5 \sigma)$ & $<-1.4$ \\
\hline
\end{tabular}
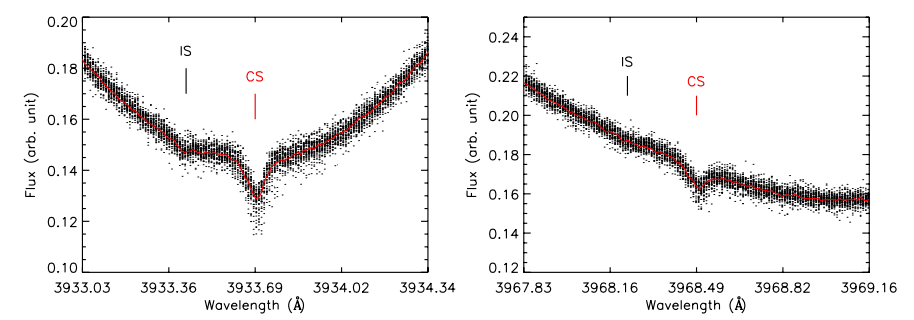

Fig. 1. Ca II reference spectrum of HD 172555 (red line) in the Ca II K and $\mathrm{H}$ lines (left and right panels, respectively). All of the 129 spectra are represented by tiny black dots. The position of the interstellar (IS) and circumstellar (CS) absorptions are identified by vertical ticks. Wavelengths are expressed in the heliocentric rest frame.

presence of a large number of FEB absorptions in most of the spectra (Kiefer et al., in prep.). The resulting reference spectrum for HD 172555 is plotted in Fig. 1, where it is compared to the whole set of flux data points.

\section{Results}

\subsection{The stable gaseous component}

A first absorbing component in the Ca II appears at blueshifted velocities $\left(v \sim-19 \mathrm{~km} \mathrm{~s}^{-1}\right)$; it is also clearly detected in the Na I D2 and D1 lines (Table 1). A second feature is seen in the Ca II domain; this is the strongest feature in the spectrum of HD 172555. It is located at the star radial velocity $\left(\sim 2 \mathrm{~km} \mathrm{~s}^{-1}\right)$; no feature is detected at the corresponding wavelengths in the Na I domain (Fig. 2).

The first feature at $-19 \mathrm{~km} \mathrm{~s}^{-1}$ presents an equivalent width ratio $E q W\left(\mathrm{NaI} D_{2}\right) / E q W(\mathrm{CaII} K) \sim 1$ which is typical of the local interstellar medium (Welsh et al. 2010). In the line of sight of HD $172555\left(l=330.6^{\circ} ; b=-23.8^{\circ}\right)$, an absorption by the $\mathrm{G}$ cloud of the local interstellar medium is expected at $-17 \mathrm{~km} \mathrm{~s}^{-1}$ (Lallement \& Bertin 1992; Redfield \& Linsky 2008). We thus conclude that this stable component is most likely due to the $\mathrm{G}$ cloud in the interstellar medium.

On the contrary, the second feature is (i) seen at the stellar radial velocity; (ii) the strongest absorption in the $\mathrm{Ca}$ II lines; and (iii) not seen in Na I. This non-detection yields a $5 \sigma$ upper limit for the equivalent widths ratio of $E q W\left(\mathrm{NaI} D_{2}\right) / E q W(\mathrm{Ca}$ II $K)<0.04$. This upper limit is much lower than typical values seen in the local interstellar medium where it is usually greater than 0.1 (see, e.g., Welsh et al. 2010). Keeping in mind that the $\mathrm{Na} / \mathrm{Ca}$ II ratio for the $\beta$ Pic $\mathrm{CS}$ gas disk is also anomalously low compared to IS standard, we conclude that the absorption detected in Ca II at the star's radial velocity is most likely related to the CS gas of the HD 172555 disk.

\subsection{Falling evaporating bodies}

A quick look at the HARPS HD 172555 spectra immediately reveals the presence of variations in the CS Ca II line seen at the star radial velocity and identified as due to CS gas (Sect. 3.1). These variations are seen at four different epochs over a total of
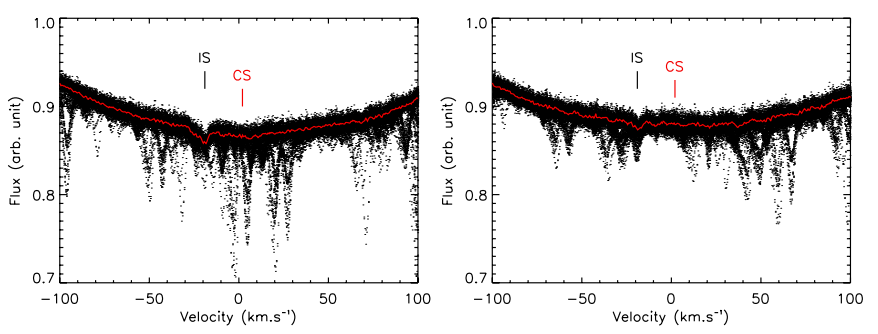

Fig. 2. Na I reference spectrum of HD 172555 (red line) in the Na I D2 and D1 lines (left and right panels, respectively) using the same caption as that of Fig. 1. Velocities are expressed in the heliocentric rest frame. No significant $\mathrm{Na}$ I absorption feature appears at the star's radial velocity $\left(v \sim 2 \mathrm{~km} \mathrm{~s}^{-1}\right)$, suggesting the CS origin of the absorption detected in the $\mathrm{Ca}$ II lines at this velocity. Conversely, the strongest absorption in $\mathrm{NaI}$ is seen at $v \sim-19 \mathrm{~km} \mathrm{~s}^{-1}$ and is most likely of interstellar origin. The narrow absorption lines seen in most of the spectra (black dots) and not in the calculated reference spectrum are due to atmospheric water.

Table 2. HARPS observations of HD 172555.

\begin{tabular}{cccccc}
\hline \hline Year & \multicolumn{2}{c}{ Date } & Nb of & Comment & Minimum \\
& duration & \\
\hline 2004 & 53147 & $(\mathrm{D} / \mathrm{M} / \mathrm{Y})$ & spectra & & \\
& 53156 & & 6 & & \\
& 53270 & $22 / 09 / 04$ & 6 & FEB & $3.5 \mathrm{~h}$ \\
\hline 2005 & 53603 & $21 / 08 / 05$ & 2 & FEB & $3 \mathrm{~min}$ \\
\hline 2006 & 53875 & & 2 & & \\
& 53880 & & 2 & & \\
& 53881 & & 4 & & \\
& 53989 & & 2 & & \\
\hline 2009 & 54942 & & 15 & & \\
& 54943 & & 10 & & \\
& 55032 & & 1 & & \\
& 55035 & & 1 & & \\
& 55148 & & 2 & & \\
\hline 2010 & 55384 & & 2 & & \\
& 55385 & $08 / 07 / 10$ & 2 & FEB & $3.9 \mathrm{~h}$ \\
\hline 2011 & 55723 & $11 / 06 / 11$ & 31 & FEB & \\
& 55724 & & 2 & & \\
& 55725 & & 2 & & \\
& 55726 & & 4 & & \\
& 55763 & & 2 & & \\
& 55764 & & 2 & & \\
& 55765 & & 26 & & \\
\hline
\end{tabular}

Notes. Minimum duration is the time separating the event's first and last detections, i.e. the upper and lower bounds to the comet's transit ingress and egress times, respectively.

22 nights of observations (Table 2). They always show up as additional absorptions over the main CS absorption, and their detection in both the Ca II-K and Ca II-H lines confirms that they are true additional absorptions to the quiet spectrum (Fig. 3).

These variations have time scales that are probably shorter than one day since, as can be seen in Table 2, their occurrence is quite rare and at least one event, on 11 June 2011, is not observed the next day; a similar example also occurred on the 8 July 2010 , but was not seen the night before. With such a low occurrence 
F. Kiefer et al.: Exocomets in the circumstellar gas disk of HD 172555
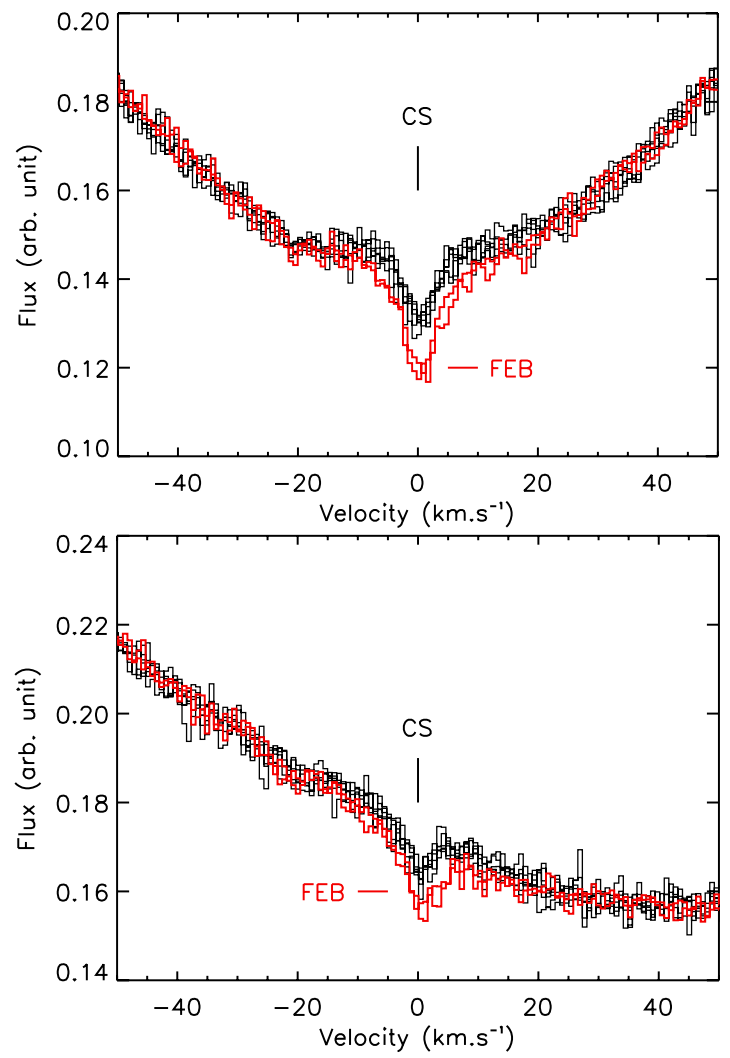

Fig. 3. HD 172555 spectra of 2004 in the Ca II K line (top panel) and $\mathrm{H}$ line (bottom panel). Two typical spectra on 22 September 2004 separated by a 6 min-time interval (red lines) clearly show the presence of an additional absorption at the star's radial velocity in comparison to spectra obtained 100 days earlier (black lines). Velocities are given in the star's rest frame.

rate, detecting an event that lasted several days just before it disappears or appears would be very fortuitous.

These variations thus share many common characteristics with those typically detected in $\beta$ Pic spectra and identified as transits of exocomets (or FEBs). To quantify the characteristics of these FEBs features, we normalized the Ca II spectra by dividing the observed spectra by the reference spectrum. Then the absorption features are fitted by a toy-model of the absorbing gaseous cloud given by

$\varepsilon(\lambda)=1-\alpha\left(1-\mathrm{e}^{-\tau(\lambda)}\right)$,

where $\alpha$ is the fraction of the stellar surface $\Sigma_{\star}$ covered by the cloud with an area $\Sigma_{\mathrm{c}}\left(\alpha=\Sigma_{\mathrm{c}} / \Sigma_{\star} \leq 1\right)$. The optical depth $\tau(\lambda)$ at the wavelength $\lambda$ is given by

$\tau(\lambda)=A \mathrm{e}^{-\frac{\left(\lambda-\lambda_{0}\right)^{2}}{2 \sigma^{2}}}$,

where the absorption depth $A$ depends on the absorbing medium density and thickness, and the line oscillator strength. Because the oscillator strength of the Ca II K line is twice that of the $\mathrm{Ca}$ II $\mathrm{H}$ line, the absorption depths in these lines follow the relationship $A_{\mathrm{K}}=2 A_{\mathrm{H}}$.

The normalized spectra of the four epochs with positive detection of FEBs are plotted in Figs. 4-7. To increase the signalto-noise ratio, we averaged the spectra taken over 30 min-time intervals. The results of the fits are given in Table 3 .

The FEBs are mostly seen at the star radial velocity, except for the FEB on 21 August 2005 with a redshifted velocity of $\sim 13 \mathrm{~km} \mathrm{~s}^{-1}$ (Fig. 5). The most significant events on

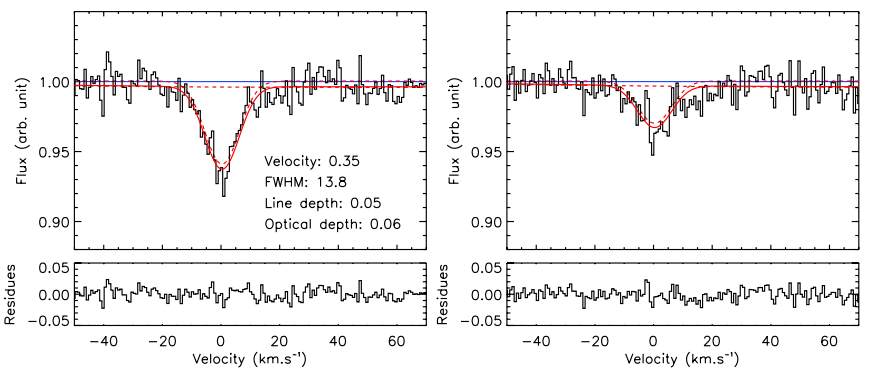

Fig. 4. Ca II normalized spectrum of the FEB detected on 22 September 2004 (MJD 53 269.996). The Ca II-K and Ca II-H lines are plotted in the left and right panels, respectively. The fits to the data are plotted with the red lines. The bottom panels show the residuals of the fits.

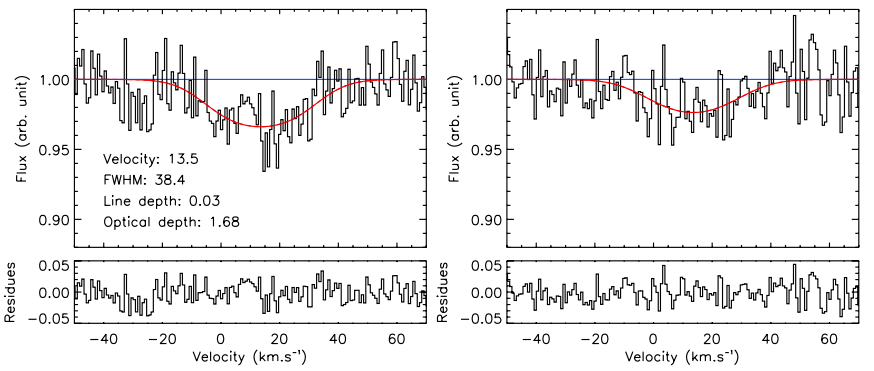

Fig. 5. Ca II normalized spectrum of the FEB detected on 21 August 2005 (MJD 53 603.145), as in Fig. 4.

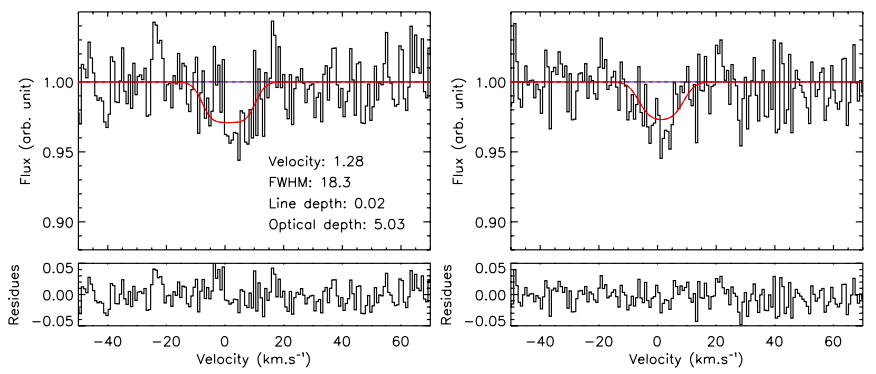

Fig. 6. Ca II normalized spectrum of the FEB detected on 08 July 2010 (MJD 55 385.285), as in Fig. 4.

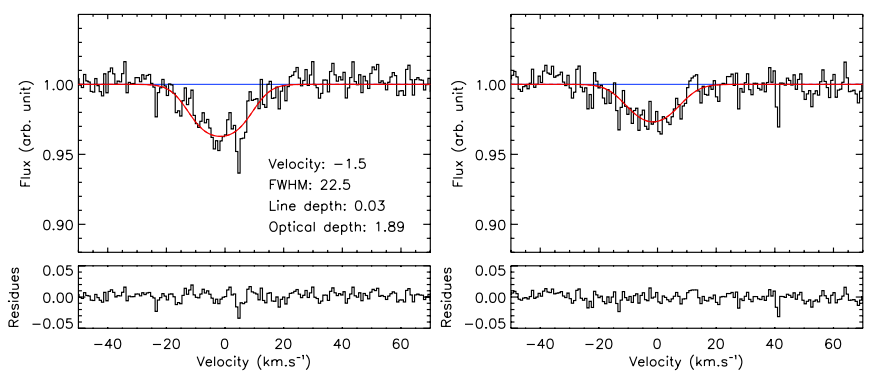

Fig. 7. Ca II normalized spectrum of the FEB detected on 11 June 2011 (MJD 55 723.248), as in Fig. 4.

22 September 2004 and 11 June 2011 have very different cloud characteristics, as often observed in $\beta$ Pic (Lagrange et al. 1992). On 22 September 2004 the absorption in the Ca II-K line is about two times deeper than in the Ca II-H line (Fig. 4), in agreement with the ratio of the lines' oscillator strengths. This is easily explained if the features are produced by large translucent clouds covering the whole surface of the star during the transit. On 11 June 2011 the absorption in the Ca II-H line is almost as deep as in the $\mathrm{K}$ line (Fig. 7); this can be explained only if it is produced by an optically thick cloud, covering only a fraction of the star surface $(\alpha \sim 0.04<1)$. The ratio of the lines can be 
Table 3. Fit parameters with 1-sigma error bars.

\begin{tabular}{|c|c|c|c|c|c|c|}
\hline \multicolumn{2}{|c|}{ Date } & \multirow[t]{2}{*}{$\mathrm{K}$ line depth } & \multirow{2}{*}{$\begin{array}{l}\text { Velocity } \\
\left(\mathrm{km} \mathrm{s}^{-1}\right)\end{array}$} & \multirow{2}{*}{$\begin{array}{c}F W H M \\
\left(\mathrm{~km} \mathrm{~s}^{-1}\right)\end{array}$} & \multirow{2}{*}{$\begin{array}{c}\text { Surface ratio } \\
\alpha\end{array}$} & \multirow[t]{2}{*}{ Optical depth } \\
\hline (MJD) & $(\mathrm{D} / \mathrm{M} / \mathrm{Y})$ & & & & & \\
\hline 53269.996 & $22 / 09 / 04$ & $0.059 \pm 0.003$ & $0.35 \pm 0.37$ & $13.9 \pm 0.9$ & $\gtrsim 0.9$ & $0.061 \pm 0.003$ \\
\hline 53270.134 & $22 / 09 / 04$ & $0.072 \pm 0.006$ & $2.3 \pm 0.5$ & $19.5 \pm 1.2$ & $\gtrsim 0.84$ & $0.075 \pm 0.006$ \\
\hline 53603.145 & $21 / 08 / 05$ & $0.034 \pm 0.004$ & $13.5 \pm 1.5$ & $38.4 \pm 9.7$ & $0.04_{-0.01}^{+0.04}$ & $1.69 \pm 1.05$ \\
\hline 55385.285 & $08 / 07 / 10$ & $0.029 \pm 0.006$ & $1.26 \pm 1.07$ & $18.4_{-101}^{+7.9}$ & $\gtrsim 0.024$ & $\lesssim 10.3$ \\
\hline 55723.248 & $11 / 06 / 11$ & $0.037 \pm 0.002$ & $-1.6 \pm 0.5$ & $22.5 \pm 3.2$ & $0.04 \pm 0.01$ & $1.90 \pm 0.63$ \\
\hline 55723.280 & $11 / 06 / 11$ & $0.032 \pm 0.002$ & $-2.44 \pm 0.51$ & $24.9 \pm 3.3$ & $0.04 \pm 0.01$ & $1.48 \pm 0.51$ \\
\hline 55723.309 & $11 / 06 / 11$ & $0.030 \pm 0.003$ & $-3.24 \pm 0.73$ & $24.2 \pm 4.7$ & $0.04_{-0.01}^{+0.02}$ & $1.59 \pm 0.78$ \\
\hline
\end{tabular}

Notes. The upper and lower limits are given at the 1-sigma level.

equal to one if they are produced by optically thick clouds (see discussion in Lecavelier des Etangs et al. 1997).

Although the two events on 21 August 2005 and 08 July 2010 are noisy, their detection remains statistically significant, thanks to the simultaneous presence of the absorption both in the $\mathrm{Ca}$ II-K and Ca II-H lines. For comparison, the very nice signal detected on 11 June 2011 was actually obtained by averaging around 10 spectra similar to the ones seen on 21 August 2005 and 08 July 2010. Because of the noise, our line-finder procedure detected only one statistically significant FEB on 21 August 2005 (Fig. 5); however, the plot suggests the possibility of two absorbing components: one at about the star's radial velocity and another at $20 \mathrm{~km} \mathrm{~s}^{-1}$. This would explain the particularly large FWHM found by fitting the line with a single component (Table 3).

Table 3 also shows that the small thick clouds are found together with large FWHM. In the framework of the FEB scenario, this is interpreted by clouds transiting at very short distances to the star (a few stellar radii) for which large orbital velocity dispersion within the FEB coma yields large Doppler broadening of the lines. Meanwhile, high radiation pressure compressed the extension of the exocomets' coma into very small size (Beust et al. 1991, 1996). These similarities with the $\beta$ Pic FEBs strengthen the conclusion that we are witnessing the transits of exocomets in the young circumstellar disk of HD 172555, like in $\beta$ Pic.

\section{Discussion}

Considering that HD 172555 and $\beta$ Pic have similar ages and spectral types, and that both stars are surrounded by gaseous and dusty circumstellar disks, the detection of (i) an absorption with an anomalously low $\mathrm{Na} \mathrm{I/Ca} \mathrm{II} \mathrm{ratio} \mathrm{at} \mathrm{the} \mathrm{star} \mathrm{radial} \mathrm{velocity;}$ and (ii) four events of variable absorptions in the Ca II line, it is very likely that we are witnessing in HD 172555 a similar phenomenon to the one in $\beta$ Pic explained by the transit of cometlike evaporating bodies.

Although the statistics is still poor for HD 172555, we can already note a difference in Doppler velocities between the $\beta$ Pic and HD 172555 FEBs. While in $\beta$ Pic most of the FEB signatures appear to be redshifted up to a few hundreds of $\mathrm{km} / \mathrm{s}$ (see review in Vidal-Madjar et al. 1998), the FEBs of HD 172555 show up nearly at the star's radial velocity within $\pm 10 \mathrm{~km} \mathrm{~s}^{-1}$. Importantly, this is not in contradiction with the exocomet (FEB) scenario, where the velocities of the sporadic absorptions are defined by the projection on the line of sight of the comet's orbital velocity. Thus, redshifted and blueshifted absorptions correspond to FEBs transiting before and after the periastron, respectively. In HD 172555, the FEBs at low radial velocity must be transiting near their periastron (see Beust et al. 1991 for the simulation of this configuration).

The detection of exocomets' transits raised the issue of the inclination of the planetary system relative to the line of sight.
Beust \& Valiron (2007) have shown that most FEBs would remain spectroscopically undetected if the $\beta$ Pic disk was inclined by more that a few tens of degrees. This also applies to HD 172555 if the dynamical origin of the FEBs is the same. Thus, the present detection of both the FEBs and the stable Ca II absorption argues that as $\beta$ Pic the HD 172555 debris disk is probably close to edge-on. This remains consistent with the recent IR data constraining its inclination to be $I>47^{\circ}$ (Smith et al. 2012) and with the large $v \sin i$ value $\sim 116 \mathrm{~km} \mathrm{~s}^{-1}$ of this A7V star.

The spectroscopic detection of transiting exocomets has already been made in a few cases other than $\beta$ Pic, for HR 10 (Lagrange et al. 1990), and for HD 21620, HD 42111, HD 110411, and HD 145964 (Welsh \& Montgomery 2013). Here for the first time, we have presented the detection of a CS absorption feature associated with variable features detected simultaneously in the two Ca II lines, which ensure the veracity of their detection.

Now further monitoring of HD 172555 is needed to obtain additionnal data and to constrain characteristics of these exocomets orbiting within a young planetary system. In analogy with $\beta$ Pic, observations of signatures of these exocomets in other spectral lines at different wavelengths can be anticipated with future observations.

Acknowledgements. This work has been supported by an award from the Fondation Simone et Cino Del Duca. The authors also acknowledge the support from the French Agence Nationale de la Recherche (ANR), under programs ANR-12-BS05-0012 Exo-Atmos and ANR-2010 BLAN-0505-01 (EXOZODI).

\section{References}

Beust, H., \& Valiron, P. 2007, A\&A, 466, 201

Beust, H., Vidal-Madjar, A., Ferlet, R., \& Lagrange-Henri, A. M. 1991, A\&A, 241,488

Beust, H., Lagrange, A.-M., Plazy, F., \& Mouillet, D. 1996, A\&A, 310, 181

Cote, J. 1987, A\&A, 181, 77

Ferlet, R., Vidal-Madjar, A., \& Hobbs, L. M. 1987, A\&A, 185, 267

Johnson, B. C., Lisse, C. M., Chen, C. H., et al. 2012, ApJ, 761, 45

Lagrange-Henri, A. M., Beust, H., Ferlet, R., Vidal-Madjar, A., \& Hobbs, L. M. 1990, A\&A, 227, L13

Lagrange-Henri, A. M., Gosset, E., Beust, H., Ferlet, R., \& Vidal-Madjar, A. 1992, A\&A, 264, 637

Lallement, R., \& Bertin, P. 1992, A\&A, 266, 479

Lecavelier des Etangs, A., Deleuil, M., Vidal-Madjar, A., et al. 1997, A\&A, 325, 228

Lisse, C. M., Chen, C. H., Wyatt, M. C., et al. 2009, ApJ, 701, 2019

Quanz, S. P., Kenworthy, M. A., Meyer, M. R., Girard, J. H. V., \& Kasper, M. 2011, ApJ, 736, L32

Redfield, S., \& Linsky, J. L. 2008, ApJ, 673, 283

Riviere-Marichalar, P., Barrado, D., Augereau, J.-C., et al. 2012, A\&A, 546, L8 Smith, R., Wyatt, M. C., \& Haniff, C. A. 2012, MNRAS, 422, 2560

Vidal-Madjar, A., Ferlet, R., Hobbs, L. M., Gry, C., \& Albert, C. E. 1986, A\&A, 167,325

Vidal-Madjar, A., Lecavelier des Etangs, A., \& Ferlet, R. 1998, Planet. Space Sci., 46, 629

Welsh, B. Y., \& Montgomery, S. 2013, PASP, 125, 759

Welsh, B. Y., Lallement, R., Vergely, J.-L., \& Raimond, S. 2010, A\&A, 510, A54 